\title{
Endoplasmic reticulum stress/XBP1 promotes airway mucin secretion under the influence of neutrophil elastase
}

\author{
XIAOYAN XU ${ }^{1,2}$, QI LI $^{3,4 *}$, LIANG $\mathrm{LI}^{3}$, MAN ZENG $^{3}, \mathrm{XIANGDONG} \mathrm{ZHOU}^{3,4}$ and ZHAOZHONG CHENG ${ }^{1 *}$ \\ ${ }^{1}$ Department of Respiratory Medicine, The Affiliated Hospital of Qingdao University, Shinan, Qingdao 266071; \\ ${ }^{2}$ Department of Respiratory Medicine, Zhucheng People's Hospital, Zhucheng, Shandong 262200; \\ ${ }^{3}$ Department of Respiratory Medicine, The First Affiliated Hospital, Hainan Medical University, Haikou, \\ Hainan 570102; ${ }^{4}$ Key Laboratory of Emergency and Trauma of Ministry of Education, \\ Hainan Medical University, Haikou, Hainan 579199, P.R. China
}

Received May 3, 2020; Accepted February 17, 2021

DOI: $10.3892 / \mathrm{ijmm} .2021 .4914$

\begin{abstract}
Endoplasmic reticulum (ER) stress is an important reaction of airway epithelial cells in response to various stimuli, and may also be involved in the mucin secretion process. In the present study, the effect of ER stress on neutrophil elastase (NE)-induced mucin (MUC)5AC production in human airway epithelial cells was explored. 16HBE14o-airway epithelial cells were cultured and pre-treated with the reactive oxygen species (ROS) inhibitor, $\mathrm{N}$-acetylcysteine (NAC), or the ER stress chemical inhibitor, 4-phenylbutyric acid (4-PBA), or the cells were transfected with inositol-requiring kinase $1 \alpha$ (IRE1 $\alpha$ ) small interfering RNA (siRNA) or X-box-binding protein 1 (XBP1) siRNA, respectively, and subsequently incubated with NE. The results obtained revealed that $\mathrm{NE}$ increased ROS production in the 16HBE14o-cells, with marked increases in the levels of ER stress-associated proteins, such as glucose-regulated protein 78 (GRP78), activating transcription factor 6 (ATF6), phosphorylated protein kinase R-like endoplasmic reticulum kinase (pPERK) and phosphorylated (p)IRE1 $\alpha$. The protein
\end{abstract}

Correspondence to: Professor Zhaozhong Cheng, Department of Respiratory Medicine, The Affiliated Hospital of Qingdao University, 16 Jiangsu Road, Shinan, Qingdao 266071, P.R. China E-mail: 13791928866@163.com

Dr Qi Li, Department of Respiratory Medicine, The First Affiliated Hospital, Hainan Medical University, 31 Longhua Road, Longhua, Haikou, Hainan 570102, P.R. China

E-mail: lqlq198210@sina.com

Abbreviations: ATF6, activating transcription factor 6; 4-PBA, 4-phenylbutyric acid; ER, endoplasmic reticulum; GRP78, glucose-regulated protein 78; IRE1 $\alpha$, inositol-requiring kinase $1 \alpha$; MUC, mucin; NE, neutrophil elastase; NAC, N-acetylcysteine; PERK, protein kinase R-like endoplasmic reticulum kinase; UPR, unfolded protein response; XBP1, X-box-binding protein 1

Key words: endoplasmic reticulum stress, mucin 5AC, reactive oxygen species, inositol-requiring kinase $1 \alpha$, X-box-binding protein 1 , respiratory inflammation and mRNA levels of spliced XBP1 were also increased, and the level of MUC5AC protein was notably increased. The ROS scavenger NAC and ER stress inhibitor 4-PBA were found to reduce ER stress-associated protein expression and MUC5AC production and secretion. Further analyses revealed that MUC5AC secretion was also attenuated by IRE1 $\alpha$ and XBP1 siRNAs, accompanied by a decreased mRNA expression of spliced XBP1. Taken together, these results demonstrate that NE induces ER stress by promoting ROS production in 16HBE14o-airway epithelial cells, leading to increases in MUC5AC protein production and secretion via the IRE1 $\alpha$ and XBP1 signaling pathways.

\section{Introduction}

Mucin (MUC)5AC is a major component of gel-forming mucus in human airways, which is understood to contribute to various functions, ranging from lubrication to hydration. Mucins, including MUC5AC, are secreted in the airways both at a low baseline rate and at a high, stimulated rate. Under healthy conditions, the constant baseline level of mucin secretion supports the steady-state mucociliary clearance of inhaled particles and pathogens that enter the airways during breathing (1). During the progression of chronic airway diseases, particularly during acute exacerbations, massive levels of acidic viscous mucin have been shown to be produced in a rapid burst phase $(2,3)$. Once stimulated with mucus-promoting factors, a significant and rapid increase in both MUC5AC mRNA and protein expression in goblet cells results in an excessive accumulation of unfolded MUC5AC protein in the endoplasmic reticulum (ER) lumen. Previous studies have focused predominantly on the molecular mechanisms underlying MUC5AC gene transcription and protein synthesis $(4,5)$. However, the mechanisms through which the protein load capacity of the ER may be improved, and those through which protein folding and the handling efficiency of the ER may be increased, remain unclear; thus, further experiments are required to elucidate the underlying mechanisms.

An excessive level of mucin synthesis results in an accumulation of misfolded proteins in the ER, a condition termed as 'ER stress', which triggers a compensatory cellular 
response termed as the 'unfolded protein response' (UPR) (6). The aim of the UPR is to reverse ER stress by enhancing the protein secretion ability of the ER, as well as increasing the capacity of the ER for protein folding and processing. However, whether factors promoting MUC5AC production are able to induce ER stress, and the effect of ER stress-signaling pathways on the regulation of the production of airway MUC5AC, have yet to be elucidated. It has been reported that the overproduction of interleukin (IL)-13 and allergen-induced MUC5AC is partly dependent on ER stress (7); however, the underlying molecular signaling mechanisms have not yet been fully elucidated. Previous studies have demonstrated that the ER-stress transducer, inositol-requiring kinase $1 \alpha$ (IRE1 $\alpha$ ), is required for normal secretory protein metabolism and cytokine production $(8,9)$.

In the present study, neutrophil elastase (NE) was selected to induce MUC5AC mRNA and protein expression. The aim of the present study was to investigate the role of ER stress in NE-regulated MUC5AC expression, ultimately to better understand the mechanisms of airway mucus secretion. The results obtained may aid in the identification of therapeutic strategies with which to alleviate airway mucus hypersecretion.

\section{Materials and methods}

Cells and reagents. The immortalized human bronchial epithelial cell line, 16HBE140-, was provided by Procell Life Science \& Technology. Small interfering RNA (siRNA) for IRE1 $\alpha$, X-box-binding protein 1 (XBP1) and control siRNA were obtained from Santa Cruz Biotechnology, Inc. The IRE1 $\alpha$ siRNA sequence was: GGACGUGAGCGACAGAAU Adtdt and the XBP1 siRNA sequence was: GCCUGUCUG UACUUCAUUCtt. Mouse anti-human $\beta$-actin monoclonal antibody (cat. no. sc-517582), as well as goat anti-rabbit IgG-HRP (cat. no. sc-2004) and goat anti-mouse IgG-HRP (cat. no. sc-2005) antibodies were purchased from Santa Cruz Biotechnology, Inc. Rabbit anti-human phosphorylated (p-) protein kinase R-like endoplasmic reticulum kinase (PERK) monoclonal antibody (Thr-980) (no. AP328) was obtained from the Beyotime Institute of Biotechnology. Rabbit anti-human polyclonal antibodies against glucose-regulated protein 78 (GRP78) (cat. no. ab230508), PERK (cat. no. ab77654), activating transcription factor 6 (ATF6; cat. no. ab37149), IRE1 $\alpha$ (cat. no. ab37073), p-IRE1 $\alpha$ (Ser724; cat. no. ab48187) and XBP1 \{cat. no. ab37152; note that this antibody recognizes both active spliced (XBP1s) and inactive unspliced XBP1 [XBP1(u)]\} were purchased from Abcam. The reactive oxygen species (ROS) assay and MTT cell viability kits were obtained from the Beyotime Institute of Biotechnology. The human MUC5AC ELISA kit was purchased from R\&D Systems, Inc. Mouse anti-human MUC5AC monoclonal antibody was from Neomarkers, Inc. 4-Phenylbutyric acid (4-PBA), an ER-stress inhibitor, and $\mathrm{N}$-acetylcysteine (NAC), an inhibitor of ROS, were obtained from Merck KGaA. XtremeGENE siRNA transfection reagent was obtained from Roche Diagnostics. Microplate readers were from Tecan Group, Ltd. The confocal laser scanning microscopy was purchased from Leica Microsystems $\mathrm{GmbH}$, whereas the fluorometer was from Molecular Devices, LLC.
Cell viability assay. The appropriate concentration of $\mathrm{NE}$ was determined using an MTT assay, as previously described (10). Briefly, a total of $1 \times 10^{4} / \mathrm{ml}$ cells in $200 \mu \mathrm{l}$ cell suspension was seeded into each well of a 96 -well plate. The cells were subsequently treated with $25,50,100$ or $200 \mathrm{ng} / \mathrm{ml} \mathrm{NE}$, and incubated at $37^{\circ} \mathrm{C}$ in an atmosphere of $5 \% \mathrm{CO}_{2}$. Cell viability was determined using an MTT assay, according to the manufacturer's instructions. At 8, 12, 24, 36 and $48 \mathrm{~h}$ following treatment, $20 \mu 15 \mathrm{mg} / \mathrm{ml}$ MTT were added to each well, and the cells were incubated at $37^{\circ} \mathrm{C}$ for a further $4 \mathrm{~h}$. The supernatants were subsequently discarded, and dimethyl sulfoxide was added $(150 \mu \mathrm{l} /$ well). The plate was then placed on an orbital shaker at room temperature for $15 \mathrm{~min}$. The absorbance was read at $570 \mathrm{~nm}$ using a microplate reader (sunrise F039300; Tecan Group, Ltd.).

Cell transfection. The immortalized human bronchial epithelial cell line 16HBE14o-was used in these experiments. The cells were incubated at a density of $1.5 \times 10^{5} / \mathrm{ml}$ in 24 -well plates, and cultured with $0.45 \mathrm{ml}$ serum-free RPMI-1640 in each well. siRNA transfection reagent $(5 \mu \mathrm{l})$ was diluted with $45 \mu \mathrm{l}$ serum-free medium to reach a final volume of $50 \mu$. Aliquots $(1 \mu \mathrm{g})$ of IRE1 $\alpha$ siRNA, XBP1 siRNA or control siRNA were diluted with $50 \mu \mathrm{l}$ serum-free medium. Subsequently, the diluted siRNA and transfection reagent were mixed and incubated for a further $20 \mathrm{~min}$ at room temperature. The transfection mixture was added in a drop-wise manner to each well, and vortex-mixed gently for $10 \mathrm{sec}$, followed by an incubation at room temperature for $16 \mathrm{~h}$. After carefully removing the supernatant, the cells were washed 3 times with PBS and subsequently incubated with fresh RPMI-1640 medium containing $10 \%$ fetal bovine serum, prior to further treatment with $100 \mathrm{ng} / \mathrm{ml} \mathrm{NE}$ for a further $24 \mathrm{~h}$.

Cell culture and grouping. The $16 \mathrm{HBE} 14 \mathrm{o}-\mathrm{cell}$ s were grown in RPMI-1640 medium and incubated at $37^{\circ} \mathrm{C}$ in an atmosphere of $5 \% \mathrm{CO}_{2}$ in a humidified incubator. The cells were grouped as follows: i) The control group, where cells were cultured under normal conditions without any additional interventions; ii) the NE group, where cells were treated with $100 \mathrm{ng} / \mathrm{ml} \mathrm{NE}$; iii) the NE and 4-PBA group, where cells were pre-treated with $10 \mathrm{mmol} / \mathrm{l} 4-\mathrm{PBA}$, as previously described (11), for $30 \mathrm{~min}$ prior to NE exposure; iv) the NE and NAC group, where cells were pre-treated with $3 \mathrm{mmol} / \mathrm{l} \mathrm{NAC}$, as previously described (12), for 30 min prior to NE exposure; $v$ ) the NE and control siRNA group, where cells were treated with NE following transfection with control siRNA; vi) the NE and IRE1 $\alpha$ siRNA group, where cells were treated with NE following transfection with IRE1 $\alpha$ siRNA; and vii) the NE and XBP1 siRNA group, where cells were treated with NE following transfection with XBP1 siRNA. After grouping, all the cells were incubated at $37^{\circ} \mathrm{C}$ for a further $24 \mathrm{~h}$ subsequent to further experiments being performed.

Measurement of ROS production. The 16HBE14o-cells were treated with various concentrations of NE $(25,50$ and $100 \mathrm{ng} / \mathrm{ml}$ ) for $24 \mathrm{~h}$, and the ROS detection assay kit was then used to measure intracellular oxidative stress, according to the manufacturer's instructions. The collected cells were incubated with serum-free medium-diluted DCFH-DA $(1: 1,000$; 
final concentration, $10 \mu \mathrm{mol} / 1$ ) at a density of $5 \times 10^{6} / \mathrm{ml}$ for $20 \mathrm{~min}$ in the dark at $37^{\circ} \mathrm{C}$. After washing with serum-free medium, the ROS fluorescence intensity was observed using a fluorometer (Flexstation 3; Molecular Devices, LLC).

Quantification of MUC5AC protein expression by ELISA. The cell culture supernatants and 16HBE14o-cells were collected separately. Cell lysates at multiple dilutions (1:0, 1:1, 1:2 and 1:5) were prepared with phosphate-buffered saline (PBS). MUC5AC protein expression in the culture supernatants and cytoplasm were measured following the instructions provided with the ELISA kit. Optical densities were measured at $450 \mathrm{~nm}$ (Flexstation 3; Molecular Devices, LLC), and the results are expressed as percentages of the baseline controls.

Quantification of protein expression by western blot analysis. Total protein was isolated from the cells using RIPA buffer reagent containing PMSF. Total protein was determined by BCA assay. The total protein of each sample was $40 \mu \mathrm{g}$. Protein samples were separated on a 5-10\% SDS-PAGE gel, and the separated proteins were then transferred onto a polyvinylidene difluoride (PVDF) membrane. Subsequent to being blocked with PBS containing 0.05\% Tween-20 and 5\% skimmed milk for $1 \mathrm{~h}$, the membranes were incubated for $2 \mathrm{~h}$ at room temperature with antibodies against GRP78, p-PERK, PERK, ATF6, p-IRE1 $\alpha$, IRE1 $\alpha$, XBP1 (all dilution, 1:500) and $\beta$-actin (dilution, 1:500). After washing, the membrane was incubated with an HRP-conjugated secondary antibody (dilution, 1:1,000) for $2 \mathrm{~h}$ at room temperature. Peroxidase activity was detected using an enhanced chemiluminescence (ECL) reagent (Applygen Technologies Inc.). The relative band density of the target protein compared with $\beta$-actin was quantified using Quantity One Analysis software (Bio-Rad Laboratories, Inc.).

Quantification of XBPI $m R N A$ expression by reverse transcription-quantitative PCR $(R T-q P C R)$. Total RNA was isolated from the cultured 16HBE140-cells in each group using TRIzol ${ }^{\circledR}$ reagent. The RNA samples were stored at $-20^{\circ} \mathrm{C}$ following initial quantification. RT-qPCR analysis of XBP1s mRNA levels was performed using a two-step RT-PCR kit according to the manufacturer's instructions. The specific primers for PCR were as follows: XBP1s forward, 5'-GGAGTTAAGACAGCGCTTGG-3' and reverse, 5'-GTC AATACCGCCAGAATCC-3'; and GAPDH forward, 5'-GGG AAGGTGAAGGTGGGAGTG-3' and reverse, 5'-AGCAGA GGGGGCAGAGATGAT-3'. The amplification process was performed for 40 cycles under the following conditions: Pre-denaturation at $95^{\circ} \mathrm{C}$ for $10 \mathrm{~min}$; denaturation at $95^{\circ} \mathrm{C}$ for $35 \mathrm{sec}$; annealing at $56^{\circ} \mathrm{C}$ for $55 \mathrm{sec}$, and extension at $72^{\circ} \mathrm{C}$ for $3 \mathrm{~min}$. The relative mRNA levels of XBP1s were analyzed using the $2^{-\Delta \Delta \mathrm{Cq}}$ method (13).

Quantification of MUC5AC expression by immunofluorescence assay. The 16HBE14o-cells were seeded on glass coverslips in 24-well plates at a density of $1 \times 10^{5} / \mathrm{ml}$, and fixed with $4 \%$ paraformaldehyde at room temperature for $30 \mathrm{~min}$. Subsequently, the cells were permeabilized with $0.1 \%$ Triton X-100 for 15 min and were blocked with goat serum for $45 \mathrm{~min}$. The cells were then incubated with $150 \mu \mathrm{l}$ primary monoclonal antibody against MUC5AC (mouse anti-human; dilution, 1:200) overnight at $4^{\circ} \mathrm{C}$, followed by incubation in fluorescein isothiocyanate-conjugated secondary antibody for $1 \mathrm{~h}$. Cell nuclei were stained with propidium iodide (PI) for $5 \mathrm{~min}$, and images were captured using a fluorescence confocal microscope (TCS-SP2; Leica Microsystems $\mathrm{GmbH}$ ). Finally, the relative fluorescence intensity of the cells was analyzed using Image Pro Plus software 6.0.

Statistical analysis. All data are presented as the means \pm SD. Statistical analyses were performed with SPSS 17.0 software (SPSS, Inc.). All experiments were performed with at least 6 cell cultures, in duplicate or triplicate. One-way analysis of variance (ANOVA) was used followed by Bonferroni analysis. A value of $\mathrm{P}<0.05$ was considered to indicate a statistically significant difference.

\section{Results}

Cell viability upon incubation with $N E$. To select the proper concentration and treatment duration for $\mathrm{NE}$ incubation, various concentrations of $\mathrm{NE}(25,50,100$ and $200 \mathrm{ng} / \mathrm{ml})$ were used for the treatment of the cells. MTT assay was used to detect the absorbance from 8 to $36 \mathrm{~h}$ in each group. MTT assay revealed similar levels of cell viability from 8 to $36 \mathrm{~h}$ between the control group and the NE-treated groups following treatment with $25-100 \mathrm{ng} / \mathrm{ml} \mathrm{NE}$, indicating that exposure to NE within this concentration range up to $36 \mathrm{~h}$ was essentially non-toxic to the 16HBE14o-cells. However, treatment with $200 \mathrm{ng} / \mathrm{ml} \mathrm{NE}$ for $36-48 \mathrm{~h}$ evidently reduced cell viability $(\mathrm{P}<0.05)$ (Fig. 1). Therefore, $100 \mathrm{ng} / \mathrm{ml} \mathrm{NE}$ was selected as the intervention condition.

NE promotes ROS production. ER stress may be activated by ROS; therefore, the levels of ROS production in cells were detected following incubation with NE. The production of ROS in the cultured 16HBE14o-cells was detected using DCFH-DA. The results revealed a significant increase in ROS production in the NE treatment group compared with the control group. Furthermore, the production of ROS was found to increase with the increasing NE concentration; ROS production in the $100 \mathrm{ng} / \mathrm{ml} \mathrm{NE}$ treatment group was found to be markedly higher, as compared with the negative control group (Fig. 2).

ROS activates the UPR and increases the expression of ER stress-related proteins. To investigate whether NE activates the ER stress pathway, the levels of ER stress-related proteins were assayed following NE stimulation. Western blot analysis was performed to examine the p-PERK, p-IRE1 $\alpha$, ATF6 and GRP78 protein expression levels in the 16HBE14o-cells. As shown in Fig. 3, the protein expression levels of p-PERK, p-IRE1 $\alpha$, ATF6 and GRP78 were significantly increased following treatment of the cells with $100 \mathrm{ng} / \mathrm{ml} \mathrm{NE}$ (all $\mathrm{P}<0.01)$. To further explore the involvement of ROS in the NE-induced increase in the expression of ER-related proteins, the 16HBE14o-cells were pre-incubated with NAC, an inhibitor of ROS. It was found that NAC markedly attenuated the NE-induced upregulation in the protein expression of p-PERK, p-IRE1 $\alpha$, ATF6 and GRP78 (all P<0.01). These data demonstrated that the generation of ROS was primarily involved in NE-induced ER stress-associated protein production. When 


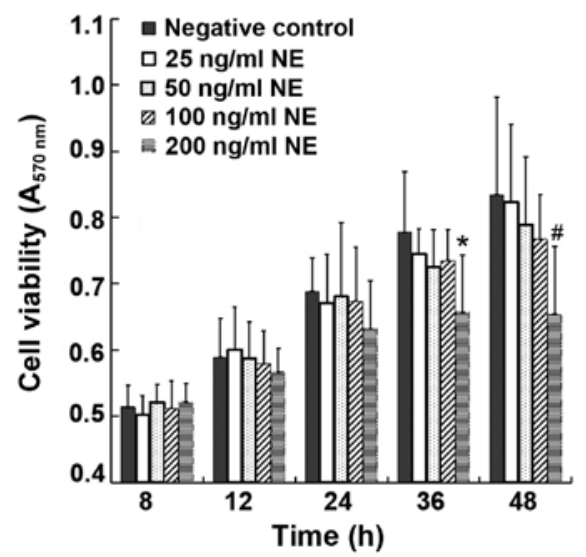

Figure 1. Proliferation of cells detected by MTT assay. Cells were exposed to various concentrations of $\mathrm{NE}$ (treatment groups were as follows: Negative control, and 25, 50, 100 and $200 \mathrm{ng} / \mathrm{ml} \mathrm{NE})$ for different periods of time (8, $12,24,36$ and $48 \mathrm{~h}$ ). Data are presented as the means $\pm \mathrm{SD}$ ( $\mathrm{n}=8$ per group). "P $<0.05$ vs. $100 \mathrm{ng} / \mathrm{ml} \mathrm{NE}$ group for $36 \mathrm{~h} ;{ }^{*} \mathrm{P}<0.01 \mathrm{vs.} 100 \mathrm{ng} / \mathrm{ml} \mathrm{NE}$ group for 48 h. NE, neutrophil elastase.

the cells were pre-treated with the ER stress inhibitor, 4-PBA, the protein expression levels of p-PERK, p-IRE1 $\alpha$, ATF6 and GRP78 were also markedly decreased, compared with the group treated with $\mathrm{NE}$ only (all $\mathrm{P}<0.01$ ).

$X B P 1 s$ expression is increased by NE stimulation, and may be suppressed by treatment with NAC and 4-PBA. To assess whether XBP1 is involved in the NE-induced process, RT-qPCR and western blot analysis were performed to examine the mRNA and protein expression levels of XBP1s in the 16HBE14o-cells. The ROS scavenger, NAC, and the ER stress inhibitor, 4-PBA, were used to verify the probable signaling factors. The results obtained revealed significant increases in the mRNA and protein levels of XBP1s in the 16HBE14o-cells incubated with $100 \mathrm{ng} / \mathrm{ml}$ NE (Figs. 4C and 5A). Moreover, such increases induced by NE were markedly attenuated by pre-treatment with either the ROS scavenger, NAC, or the ER stress inhibitor, 4-PBA (all $\mathrm{P}<0.01$; Figs. $4 \mathrm{C}$ and $5 \mathrm{~A}$ ), suggesting that NE may increase XBP1 expression, and that this may be mediated via ROS generation and ER stress activation.

$X B P 1$ expression levels are reduced following transfection with IREI $\alpha$ siRNA, XBPI siRNA. To further evaluate the role of XBP1 and whether IRE1 $\alpha$ is responsible for XBP1 activation, the cells were further transfected with IRE1 $\alpha$ siRNA, XBP1 siRNA or negative control siRNA, and subsequently stimulated with NE. The transfection efficiency was verified by detecting the protein expression levels of IRE1 $\alpha$ and XBP1 by western blot analysis following transfection. The antibody to XBP1 used in the experiment is able to recognize total XBP1 protein including both isoforms (inactive non-spliced and active spliced) of XBP1. The results obtained revealed that minimal IRE1 $\alpha$ protein was detected in the IRE1 $\alpha$ siRNA-transfected cells. In addition minimal XBP1 protein (both unspliced and spliced form) was detected in the XBP1 siRNA-transfected cells, confirming the successful transfection (Fig. 4A and B). Following NE stimulation, the XBP1s mRNA and protein expression levels were significantly decreased in the cells transfected with IRE1 $\alpha$ and XBP1

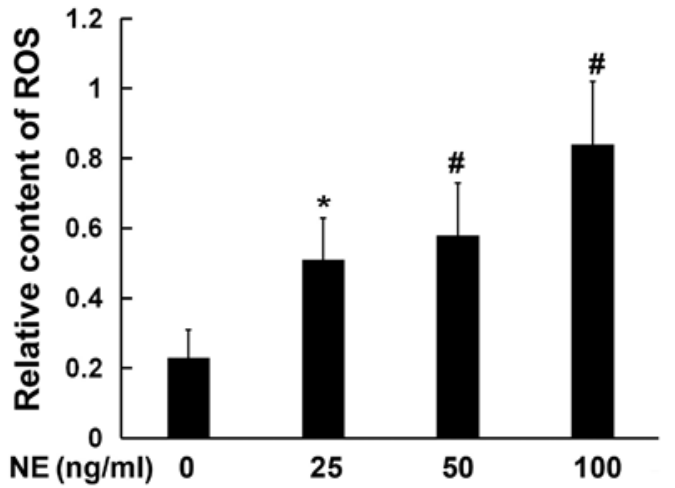

Figure 2. Effect of NE on ROS generation in 16HBE14o-cells. Cells were treated with various concentrations of NE for $24 \mathrm{~h}$, and the generation of ROS was detected using a ROS detection kit. Data are presented as the means $\pm \mathrm{SD}$ ( $\mathrm{n}=8$ per group). ${ }^{*} \mathrm{P}<0.05$ vs. control $(0 \mathrm{ng} / \mathrm{ml})$; ${ }^{\#} \mathrm{P}<0.01$ vs. control $(0 \mathrm{ng} / \mathrm{ml})$. $\mathrm{NE}$, neutrophil elastase; ROS, reactive oxygen species.

siRNA, compared with the normal control siRNA-transfected cells (all $\mathrm{P}<0.01$; Figs. 4D and 5B). These results demonstrate that IRE1 $\alpha$ may be responsible for XBP1 activation.

The IRE1 $\alpha$-XBP1 pathway is involved in NE-induced MUC5AC expression. In order to measure the mucin content during pathological conditions, the major airway mucin MUC5AC protein and mRNA expression were assayed following NE stimulation. The roles of the ROS and ER stress pathways in the process of MUC5AC production were also assessed by pre-treating the cells with NAC and 4-PBA. The results revealed that the exposure of $16 \mathrm{HBE} 140$-cells to $100 \mathrm{ng} / \mathrm{ml} \mathrm{NE}$ led to an increase in MUC5AC protein production in both the supernatant and cytoplasm, although these increases in the level of MUC5AC protein were clearly attenuated by pre-treatment with NAC or 4-PBA $(\mathrm{P}<0.01)$ (Fig. 6A), indicating that oxidative stress and ER stress may be involved in the MUC5AC production process. Moreover, to investigate whether IRE1 $\alpha$ and XBP1 participate in the MUC5AC production, the cells were transfected with IRE1 $\alpha$ siRNA or XBP1 siRNA prior to NE stimulation. The data indicated that NE-induced MUC5AC mRNA expression and protein production were significantly decreased in both the supernatant and cytoplasm $(\mathrm{P}<0.01$; Fig. $6 \mathrm{~B})$. The results of immunofluorescence assay revealed that MUC5AC staining was diminished in all the experimental groups that were pre-treated with NAC or 4-PBA, or transfected with IRE1 $\alpha$ or XBP1 siRNA, when compared with the NE-treated group $(\mathrm{P}<0.01$; Fig. 7). Taken together, these results suggest that the IRE1 $\alpha / \mathrm{XBP} 1$ pathway may be involved in NE-induced MUC5AC expression.

\section{Discussion}

In the present study, it was demonstrated that NE stimulation resulted in the activation of the ER stress-associated proteins, PERK, IRE1 $\alpha$ and ATF6, and MUC5AC mucin secretion. Treatment with the ROS scavenger, NAC, and the ER stress inhibitor, 4-PBA, led to a decrease in ER stress-associated protein expression and MUC5AC production. In addition, it was observed that MUC5AC secretion was notably reduced by the knockdown of XBP1 expression through IRE1 $\alpha$ siRNA 
A

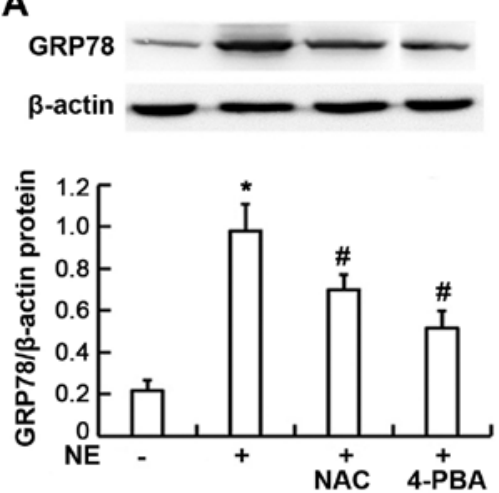

C
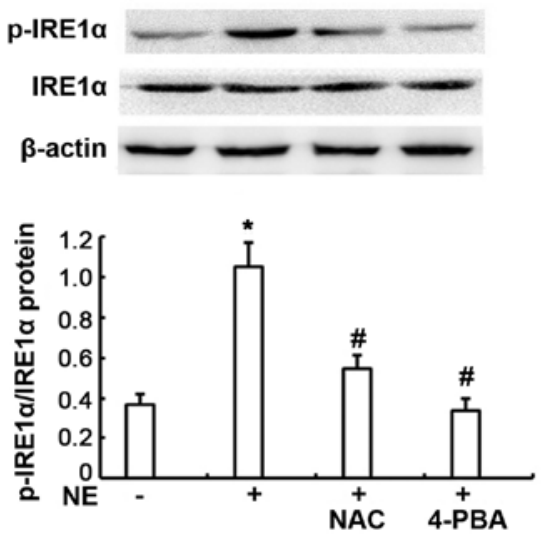

B
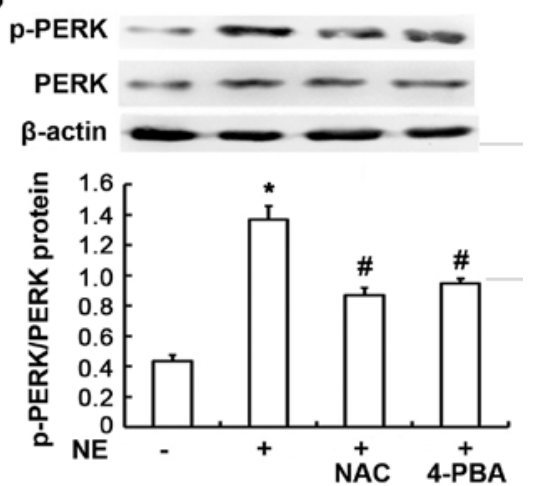

D
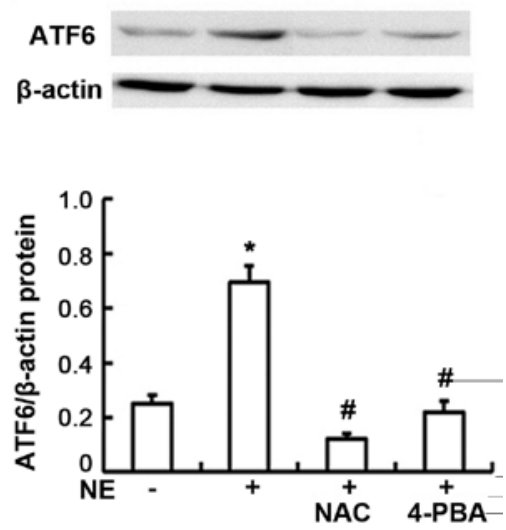

Figure 3. Expression of endoplasmic reticulum stress-related proteins in $16 \mathrm{HBE} 14 \mathrm{o}-\mathrm{cells}$. Cells were exposed to $100 \mathrm{ng} / \mathrm{ml} \mathrm{NE}$ or pre-treated with NAC and 4-PBA prior to NE exposure. GRP78, PERK, p-PERK, IRE1 $\alpha$, p-IRE1 $\alpha$, and ATF6 proteins were assayed by western blot analysis. (A) Relative GRP78 protein expression levels are presented as the ratio of GRP78 to $\beta$-actin. (B) Relative p-PERK protein expression levels are presented as the ratio of p-PERK to PERK, $\beta$-actin blots are for the loading control. (C) Relative p-IRE1 $\alpha$ protein expression levels are presented as the ratio of pIRE1 $\alpha$ to IRE1 $\alpha, \beta$-actin blots are for the loading control. (D) Relative ATF6 protein expression levels are presented as the ratio of ATF6 to $\beta$-actin. Data are presented as the means \pm SD ( $=4$ samples per group). $\mathrm{P}<0.01$ vs. negative control; ${ }^{\#} \mathrm{P}<0.01$ vs. the NE group. NE, neutrophil elastase; NAC, N-acetylcysteine; ATF6, activating transcription factor 6; 4-PBA, 4-phenylbutyric acid; GRP78, glucose-regulated protein 78; IRE1 $\alpha$, inositol-requiring kinase 1 $\alpha$; p, phosphorylated; PERK, protein kinase R-like endoplasmic reticulum kinase.

and XBP1 siRNA transfection, suggesting that ER stress signaling, particularly that involved with the IRE1 $\alpha-\mathrm{XBP} 1$ pathway, may play an important role in MUC5AC production induced by NE.

Airway mucus hypersecretion is a major pathophysiological feature in numerous patients with chronic inflammatory airway diseases. NE is a serine protease secreted by neutrophils that is highly expressed in the airway secretions of these patients (14). It is recognized as a potent and significant agonist that gives rise to MUC5AC overexpression and mucin hypersecretion $(15,16)$. A previous study demonstrated that NE-induced MUC5AC mRNA and protein expression was associated with various signaling molecular and pathways that involve the activation of protein kinase $\mathrm{C}$ (PKC), epidermal growth factor receptor (EGFR) and mitogen-activated protein kinase (MAPK) (17). In the present study, the potential role of ER stress induced by NE in the secretion of airway mucin was investigated, and the results obtained may provide novel insight into the mechanisms and regulation of airway mucin secretion; these findings may also lead to the identification of additional therapeutic strategies to alleviate airway mucus hypersecretion.

ER stress is an important reaction of airway epithelia in response to noxious stimuli. Various stimuli involving hypoxemia (18), oxidative stress (19), infection (20-22), cigarette smoke $(23,24)$ and hyperglycemia $(25)$ can disrupt ER function, resulting in the accumulation of unfolded and misfolded proteins in the ER lumen, a condition known as ER stress (26). The ER is mainly responsible for synthesizing, folding and processing secretory and transmembrane proteins correctly. Previous studies have confirmed that ER stress is involved in the occurrence and progression of airway inflammation and various pulmonary diseases, including chronic obstructive pulmonary disease (COPD), asthma and cystic fibrosis $(27,28)$.

ROS are one of the major factors causing ER stress $(29,30)$. Moreover, it is an important inducing factor in airway inflammation and mucin secretion (31). In the present study, intracellular ROS levels, as well as the protein levels of the ER chaperone, GRP78, p-PERK, p-IRE1 $\alpha$, ATF6 and XBP1s, were significantly increased by NE exposure, suggesting that NE can trigger both oxidative and ER stress. Subsequently, co-treatment with NAC, a ROS scavenger, significantly attenuated the production and activation of the above-mentioned ER stress-associated proteins. Taken together, all these results indicate that NE-induced ER stress is, at least partly, ROS-dependent.

ER stress activates a complex signaling network referred as the UPR to reduce ER stress and restore homeostasis (32). Generally, the UPR is initiated by three ER stress transducers: PERK, IRE1 and ATF6. In unstressed cells, IRE1, PERK and 
A
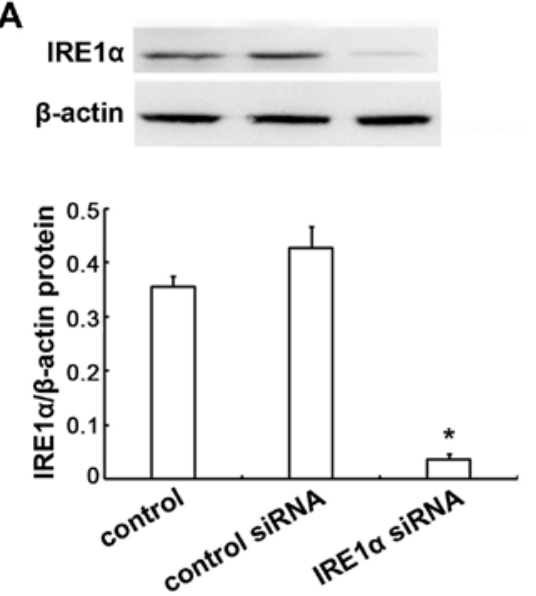

C
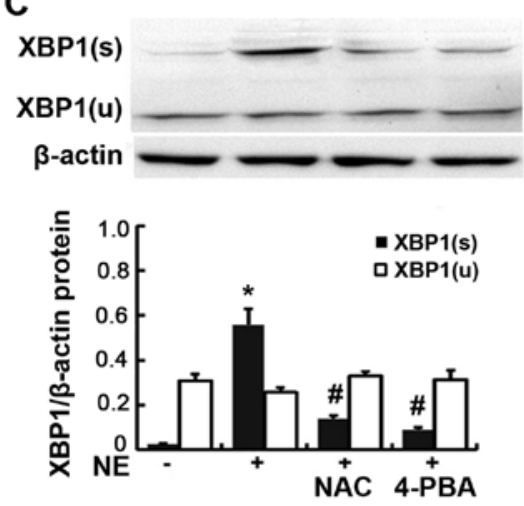

B

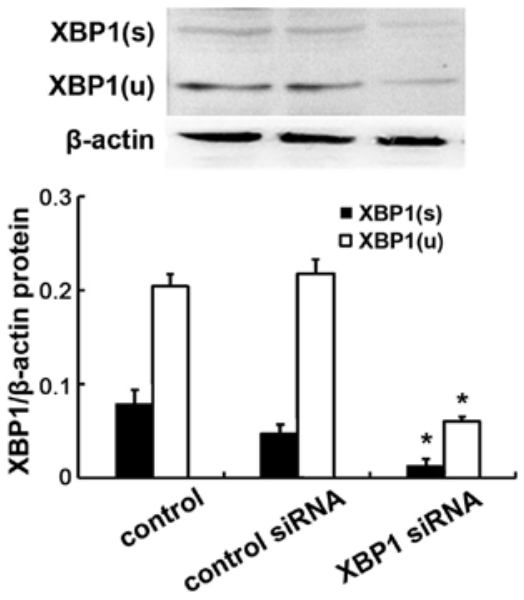

D

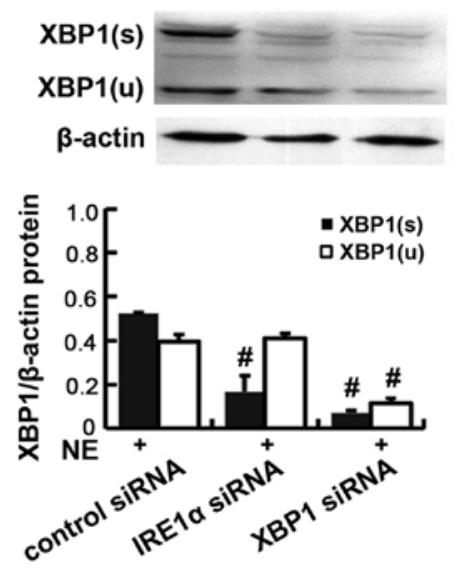

Figure 4. Protein expression of IRE1 $\alpha$ and XBP1 in 16HBE14o-cells. (A) Cells were transfected with IRE1 $\alpha$ siRNA or negative control siRNA, IRE1 $\alpha$ protein was assayed by western blot analysis. Relative IRE1 $\alpha$ protein expression levels are presented as the ratio of IRE1 $\alpha$ to $\beta$-actin. Data are presented as the means \pm SD ( $n=4$ samples per group). "P<0.01 vs. negative control and control siRNA (B) Cells were transfected with XBP1 siRNA or negative control siRNA, XBP1 protein was assayed by western blot analysis. Relative XBP1(s) protein expression levels are presented as the ratio of XBP1(s) to $\beta$-actin. Relative XBP1(u) protein expression levels are presented as the ratio of $\mathrm{XBP} 1(\mathrm{u})$ to $\beta$-actin. Data are presented as the means $\pm \mathrm{SD}$ ( $\mathrm{n}=4$ samples per group). ${ }^{*} \mathrm{P}<0.01$ vs. negative control and control siRNA. (C) Cells were exposed to $100 \mathrm{ng} / \mathrm{ml} \mathrm{NE}$ or pre-treated with NAC and 4-PBA prior to NE exposure. XBP1 protein was assayed by western blot analysis. Data are presented as the means $\pm \mathrm{SD}\left(\mathrm{n}=4\right.$ samples per group). ${ }^{*} \mathrm{P}<0.01$ vs. negative control; ${ }^{*} \mathrm{P}<0.01$ vs. the NE group. (D) Cells were transfected with IREl $\alpha$ siRNA or XBP1 siRNA, and then incubated with $100 \mathrm{ng} / \mathrm{ml} \mathrm{NE}$. Relative XBP1(s) protein expression levels are presented as the ratio of XBP1(s) to $\beta$-actin. Relative XBP1(u) protein expression levels are presented as the ratio of $\mathrm{XBP} 1(\mathrm{u})$ to $\beta$-actin. Data are presented as the means $\pm \mathrm{SD}$ ( $\mathrm{n}=4$ samples per group). ${ }^{*} \mathrm{P}<0.01$ vs. the $\mathrm{NE}+\mathrm{control}$ siRNA group. IRE1 $\alpha$, inositol-requiring kinase $1 \alpha$; NE, neutrophil elastase; NAC, N-acetylcysteine; 4-PBA, 4-phenylbutyric acid; XBP1, X-box-binding protein 1; XBP1(s), spliced XBP1; XBP1(u), unspliced XBP1.
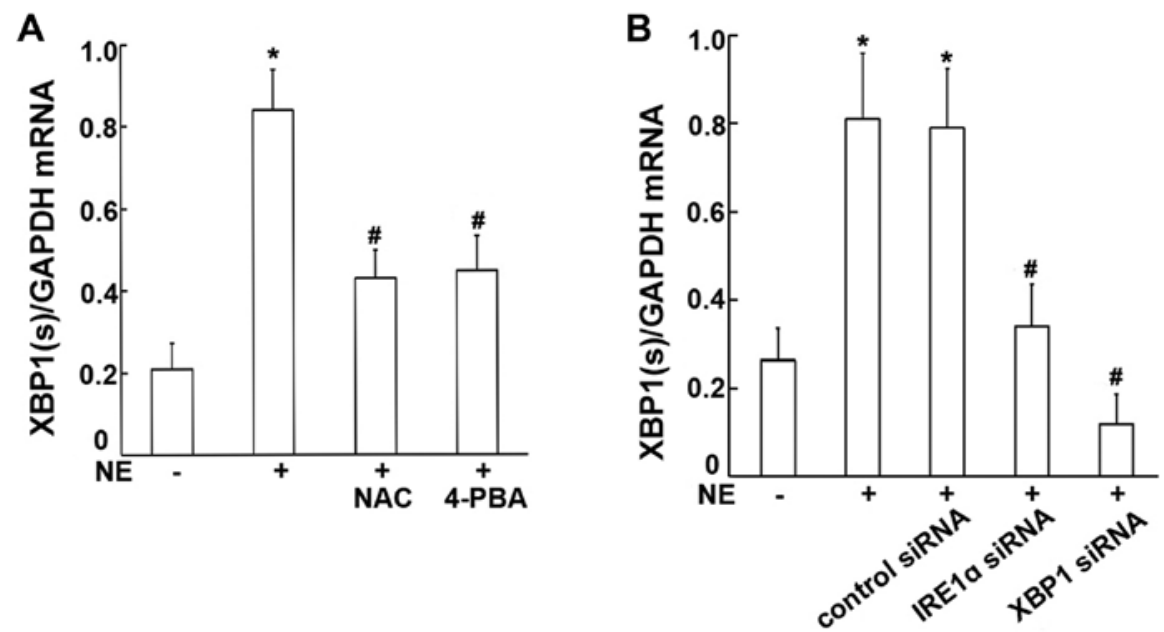

Figure 5. Expression of spliced XBP1 mRNA in 16HBE14o-cells. (A) Cells were exposed to $100 \mathrm{ng} / \mathrm{ml} \mathrm{NE}$ or pre-treated with NAC and 4-PBA prior to NE exposure. XBP1(s) was evaluated by reverse transcription-quantitative PCR. (B) Cells were transfected with IRE1 $\alpha$ siRNA or XBP1 siRNA prior to exposure to NE. Data are presented as the means $\pm \mathrm{SD}$ ( $\mathrm{n}=4$ samples per group). ${ }^{*} \mathrm{P}<0.01$ vs. control; ${ }^{*} \mathrm{P}<0.01$ vs. NE group. NE, neutrophil elastase; NAC, $N$-acetylcysteine; IRE1 $\alpha$, inositol-requiring kinase 1 $\alpha$; 4-PBA, 4-phenylbutyric acid; XBP1, X-box-binding protein 1; XBP1(s), spliced XBP1. 

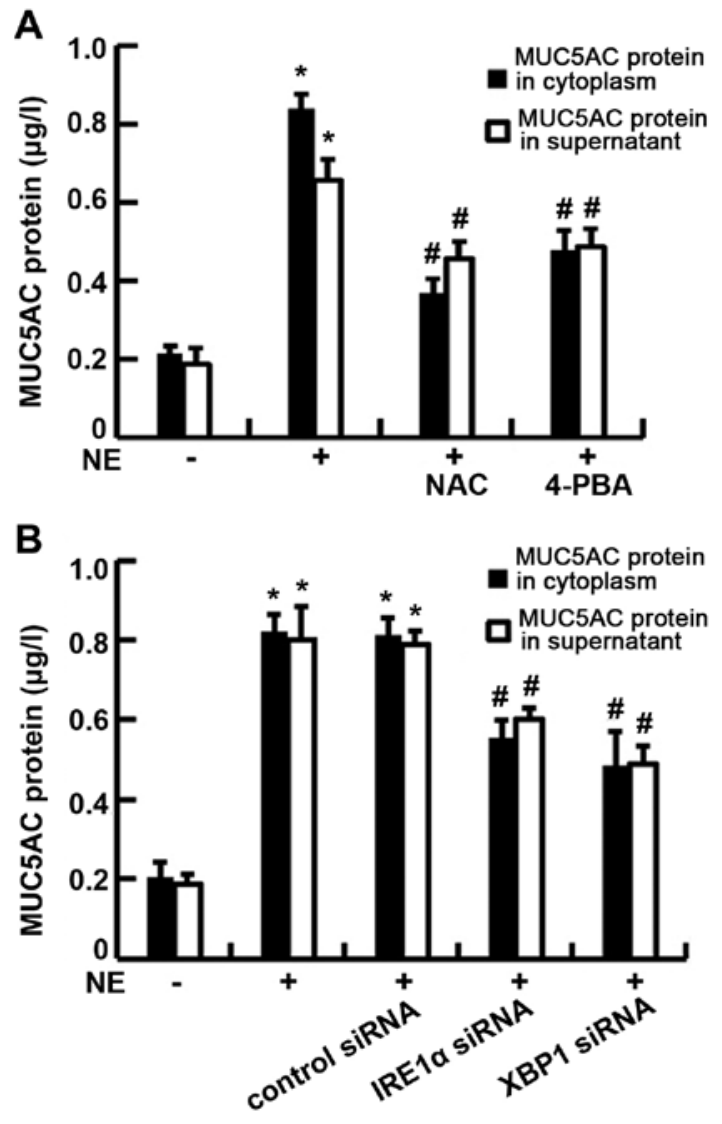

Figure 6. MUC5AC protein production in 16HBE14o-cells. (A) Cells were exposed to $100 \mathrm{ng} / \mathrm{ml} \mathrm{NE}$ or pre-treated with NAC and 4-PBA prior to NE exposure. (B) Cells were transfected with IRE1 $\alpha$ siRNA or XBP1 siRNA prior to exposure to NE. MUC5AC protein in the supernatant and in the cytoplasm were detected by ELISA. Data are presented as the means $\pm \mathrm{SD}(\mathrm{n}=4$ samples per group). ${ }^{*} \mathrm{P}<0.01$ vs. control; ${ }^{\#} \mathrm{P}<0.01$ vs. NE group. NE, neutrophil elastase; NAC, $N$-acetylcysteine; IRE1 $\alpha$, inositol-requiring kinase $1 \alpha$; 4-PBA, 4-phenylbutyric acid; XBP1, X-box-binding protein 1; MUC, mucin.

ATF6 remain in an inactive state through association with GRP78 (33). Three pathways, namely the IRE1/XBP1 pathway, the PERK/eIF2 $\alpha$ pathway and the ATF6/CHOP pathway, collectively constitute the ER-specific UPR (34). Upon the ER receiving a stress stimulus, these UPR transducers are dissociated from GRP78, and subsequently become activated. PERK is activated by the autophosphorylation of its kinase domain, which leads to the subsequent phosphorylation of eukaryotic initiation factor 2 substrate $\alpha$ (eIF2 $\alpha)$, specifically at Ser51. IRE1 $\alpha$ dimerization and autophosphorylation activates its RNase activity to splice XBP1 mRNA, thereby generating the mature spliced form of XBP1 known as XBP1s (35). It has been reported that XBP1s is a key initiator of ER stress-induced protein expression (36-38). IRE-XBP1 signaling is one of the most important pathways in ER stress, which is involved in numerous pathological processes, including tumor growth, metastasis and inflammation $(35,39)$.

IRE1 has two isoforms, IRE1 $\alpha$ and IRE1 $\beta$. IRE1 $\alpha$ is ubiquitously expressed, whereas IRE1 $\beta$ is limited to the epithelial cells, mainly at the gastrointestinal tract (40). Previous studies have demonstrated that the IRE1 $\alpha$ pathway is required for normal secretory protein metabolism (8). Park et al (41) have reported that kaempferol alleviates ovalbumin-induced airway mucus hypersecretion by inhibiting the activation of

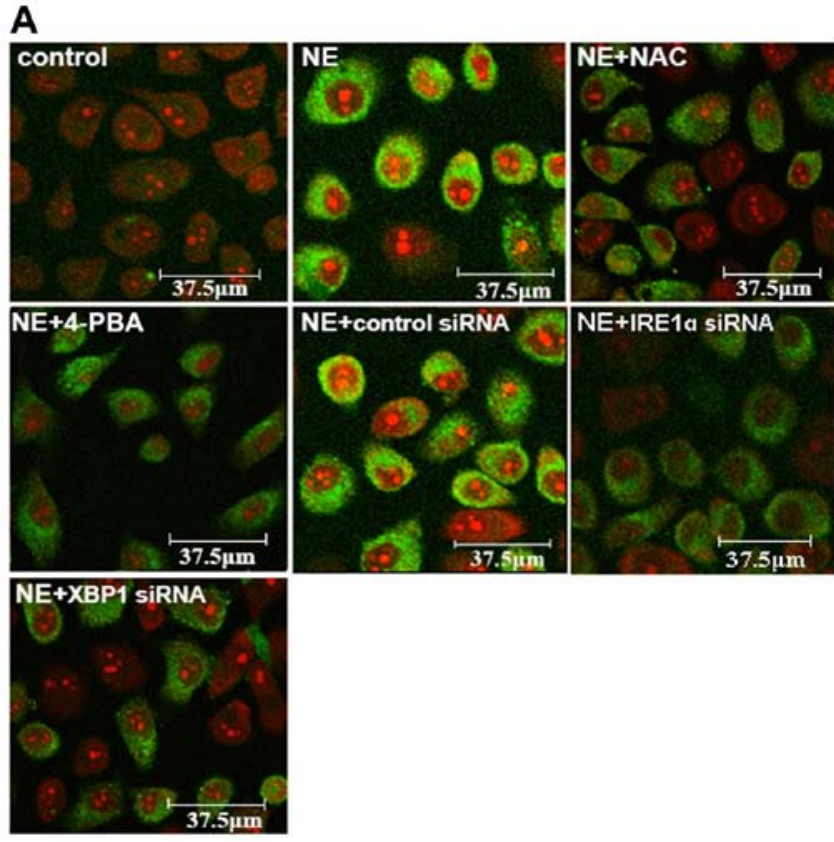

B

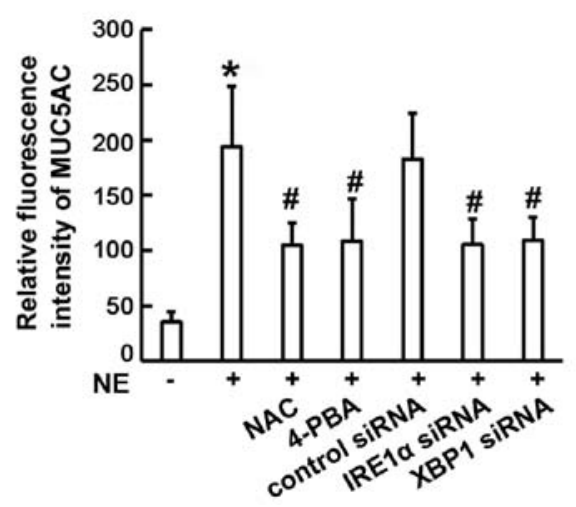

Figure 7. Intracellular MUC5AC expression in 16HBE14o-cells (A) MUC5AC was detected by immunofluorescence and confocal microscopy. Cell nuclei were stained with propidium iodide (red fluorescence). (B) The fluorescence intensities are expressed as the IOD/area. IOD/area values were calculated using Image pro-plus- 6.0 software. ${ }^{*} \mathrm{P}<0.01$ vs. control; ${ }^{\#} \mathrm{P}<0.01$ vs. NE group. NE, neutrophil elastase; NAC, $N$-acetylcysteine; 4-PBA, 4-phenylbutyric acid; MUC, mucin.

IRE1 $\alpha$ and c-Jun N-terminal kinase in human bronchial airway epithelial BEAS-2B cells. It is therefore possible to propose that an IRE1 $\alpha$-XBP1 dependent signaling cascade is involved in the synthesis and secretion of MUC5CA. To examine this hypothesis, siRNA was used to silence the IRE1 $\alpha$ and XBP1 genes. The results obtained demonstrated that both siRNAs caused a significant decrease in the mRNA and protein level of XBP1s, which was accompanied by decreases in MUC5AC synthesis and secretion. It has been reported that IRE1 $\beta$, the other specific isoform of IRE1, was able to directly induce mucin production in Calu- 3 cells by promoting XBP1 mRNA splicing-dependent transcription of AGR2, which was not mediated by an ER-stress response (6). Furthermore, IRE1 $\alpha$-dependent XBP1 mRNA splicing was found to be involved in the secretion of airway MUC5AC in the present set of experiments, suggesting a vital role of XBP1 in the IRE1 pathway.

$\mathrm{XBP} 1$ is a basic leucine zipper constitutional protein which belongs to the cAMP response element binding protein (CREB)/activating transcription factors (ATF) family (42). It 
is involved in several normal physiological processes, and is an important component of the UPR. In the UPR after ER stress, XBP1s functions as a transcription factor to initiate the expression of many target genes that regulate the unfolding of proteins (43). In a previous study, XBP1 was reported as one of the MUC5AC-associated core genes in healthy nonsmokers with high MUC5AC expression (44). In the present study, the human bronchial epithelial 16HBE14o-cell line was used, and cells that were cultured in vitro were subsequently exposed to NE. The results revealed that MUC5AC secretion was reduced following transfection with XBP1 siRNA, indicating that XBP1 may be indispensable for MUC5AC production under NE-induced ER stress. It will be interesting to examine the potential transcriptional effects of XBP1 on mucin secretion in human airways featuring other stimuli, such as smoke or fine particles.

Moreover, 4-PBA, an inhibitor of ER stress, was found to significantly decrease the expression of MUC5AC, and IRE1 $\alpha$ siRNA also markedly reduced (but did not completely inhibit) the expression of MUC5AC, indicating that other ER stress-associated proteins may also be involved in airway mucus secretion through XBP1. Indeed, all the three pathways that participate in ER stress have been shown to interact with each other. For example, XBP1 mRNA can be induced by ATF6, and ATF6 expression can be enhanced by PERK (45). A recent study reported that ATF6 and XBP1 may reduce colorectal cancer cell proliferation and stemness by activating PERK-eIF2 $\alpha$ signaling (46). The limitation of the present study is that animal experiments in vivo were not performed for verification; only the ER stress inhibitor, 4-PBA, was used while no specific inhibitors of PERK, ATF6 or IRE1 $\alpha$ were used. Among the three branches of the UPR, PERK is a most widely studied pathway with numerous functions (47). It seems difficult to act as a relatively specific blocking target. The expression of ATF6 was relatively weak in the present study, suggesting that the role of ATF6 may be less important. IRE1 $\alpha$-dependent XBP1 splicing will lead to production of the XBP1 transcription factor, this is also an important ER stress signaling pathway. MUC5AC expression in the human small airway epithelium was linked to several mucus production/secretion-related transcription factors including XBP1. Therefore, the present study mainly focused on the IRE1 $\alpha / \mathrm{XBP} 1$ pathway. However, further studies still need to be performed. The authors aim to establish animal models and select specific inhibitors or RNA interference for PERK and ATF6 in the future, in order to fully elucidate the underlying mechanism(s).

In conclusion, the present study demonstrates that ER stress is involved in NE-induced MUC5AC secretion in human airway epithelial cells, and that this is achieved mainly through the activation of the IRE1 $\alpha$ and XBP1 signaling pathways.

\section{Acknowledgements}

Not applicable.

\section{Funding}

The present study was supported by the Hainan Provincial Natural Science Fundation of China (grant nos. 820CXTD448 and ZDYF2020223) and the National Natural Science
Foundation of China (grant nos. 81860001, 31660329 and 82011530049).

\section{Availability of data and materials}

All data generated or analyzed during this study are available from the corresponding author on reasonable request.

\section{Authors' contributions}

XZ and ZC designed the study. XX, QL, LL and MZ performed the experiments. All authors contributed to the critical data analysis. All authors read and approved the final manuscript.

\section{Ethics approval and consent to participate}

Not applicable.

\section{Patient consent for publication}

Not applicable.

\section{Competing interests}

The authors declare that they have no competing interests.

\section{References}

1. Ma J, Rubin BK and Voynow JA: Mucins, mucus, and goblet cells. Chest 154: 169-176, 2018.

2. Bonser LR and Erle DJ: Airway mucus and asthma: The role of MUC5AC and MUC5B. J Clin Med 6: 112, 2017.

3. Thornton DJ, Rousseau K and McGuckin MA: Structure and function of the polymeric mucins in airways mucus. Annu Rev Physiol 70: 459-486, 2008.

4. Bae CH, Na HG, Choi YS, Song SY and Kim YD: Clusterin induces MUC5AC expression via activation of NF- $\mathrm{KB}$ in human airway epithelial cells. Clin Exp Otorhinolaryngol 11: 124-132, 2018.

5. Song KS, Yoon JH, Kim KS and Ahn DW: c-Ets1 inhibits the interaction of NF- $\kappa \mathrm{B}$ and CREB, and downregulates IL-1 $\beta$-induced MUC5A coverproduction during airway inflammation. Mucosal Immunol 5: 207-215, 2012.

6. Martino MB, Jones L, Brighton B, Ehre C, Abdulah L, Davis CW, Ron D, O'Neal WK and Ribeiro CM: The ER stress transducer IRE1 $\beta$ is required for airway epithelial mucin production. Mucosal Immunol 6: 639-654, 2013.

7. Wang X, Yang X, Li Y, Wang X, Zhang Y, Dai X, Niu B, Wu J, Yuan X, Xiong A, et al: Lyn kinase represses mucus hypersecretion by regulating IL-13-induced endoplasmic reticulum stress in asthma. EBioMedicine 15: 137-149, 2017.

8. Safra M, Ben-Hamo S, Kenyon C and Henis-Korenblit S: The IRE-1 ER stress-response pathway is required for normal secretory-protein metabolism in C. elegans. J Cell Sci 126: 4136-4146, 2013.

9. Kim S, Joe Y, Kim HJ, Kim YS Jeong SO, Pae HO, Ryter SW, Surh YJ and Chung HT: Endoplasmic reticulum stress-induced IRE1 $\alpha$ activation mediates cross-talk of GSK- $3 \beta$ and XBP1 to regulate inflammatory cytokine production. J Immunol 194: 4498-4506, 2015.

10. Deo SH, Jenkins NT, Padilla J, Parrish AR and Fadel PJ: Norepinephrine increases NADPH oxidase-derived superoxide in human peripheral bloodmonuclear cells via $\alpha$-adrenergic receptors. Am J Physiol Regul Integr Comp Physiol 305: R1124-R1132, 2013.

11. Basseri S, Lhoták S, Sharma AM and Austin RC: The chemical chaperone 4-phenylbutyrate inhibits adipogenesis by modulating the unfolded protein response. J Lipid Res 50: 2486-2501, 2009.

12. Halasi M, Wang M, Chavan TS, Gaponenko V, Hay N and Gartel AL: ROS inhibitor N-acetyl-L-cysteine antagonizes the activity of proteasome inhibitors. Biochem J 454: 201-208, 2013. 
13. Livak KJ and Schmittgen TD: Analysis of relative gene expression data using real-time quantitative PCR and the 2(-Delta Delta C(T)) method. Methods 25: 402-408, 2001

14. Kohri K, Ueki IF and Nadel JA: Neutrophil elastase induces mucin production by ligand-dependent epidermal growth factor receptor activation. Am J Physiol Lung Cell Mol Physiol 283: L531-L540, 2002.

15. Shao MX and Nadel JA: Neutrophil elastase induces MUC5AC mucin production in human airway epithelial cells via a cascade involving protein kinase $\mathrm{C}$, reactive oxygen species, and TNF-alpha-converting enzyme. J Immunol 175: 4009-4016, 2005.

16. Voynow JA, Fischer BM, Malarkey DE, Burch LH, Wong T, Longphre M, Ho SB and Foster WM: Neutrophil elastase induces mucus cell metaplasia in mouse lung. Am J Physiol Lung Cell Mol Physiol 287: L1293-L1302, 2004.

17. Park JA, Sharif AS, Shiomi T, Kobzik L, Kasahara DI, Tschumperlin DJ, Voynow J and Drazen JM: Human neutrophil elastase-mediated goblet cell metaplasia is attenuated in TACE-deficient mice. Am J Physiol Lung Cell Mol Physiol 304: L701-L707, 2013.

18. Mennerich D, Kellokumpu S and Kietzmann T: Hypoxia and reactive oxygen species as modulators of endoplasmic reticulum and golgi homeostasis. Antioxid Redox Signal 30: 113-137, 2019.

19. Zeeshan HM, Lee GH, Kim HR and Chae HJ: Endoplasmic reticulum stress and associated ROS. Int J Mol Sci 17: 327, 2016

20. Zeng M, Sang W, Chen S, Chen R, Zhang H, Xue F, Li Z, Liu Y, Gong Y, Zhang $\mathrm{H}$ and Kong X: 4-PBA inhibits LPS-induced inflammation through regulating ER stress and autophagy in acute lung injury models. Toxicol Lett 5: 271: 26-37, 2017.

21. Martinon F, Chen X, Lee AH and Glimcher LH: Toll-like receptor activation of XBP1 regulates innate immune responses in macrophages. Nat Immunol 11: 411-418, 2010.

22. Keestra-Gounder AM, Byndloss MX, Seyffert N, Young BM, Chávez-Arroyo A, Tsai AY, Cevallos SA, Winter MG, Pham OH, Tiffany CR, et al: NOD1 and NOD2 signalling links ER stress with inflammation. Nature 532: 394-397, 2016.

23. Wang Y, Wu ZZ and Wang W: Inhibition of endoplasmic reticulum stress alleviates cigarette smoke-induced airway inflammation and emphysema. Oncotarget 8: 77685-77695, 2017.

24. Kunchithapautham K, Atkinson C and Rohrer B: Smoke exposure causes endoplasmic reticulum stress and lipid accumulation in retinal pigment epithelium through oxidative stress and complement activation. J Biol Chem 289: 14534-14546, 2014.

25. Li XZ, Xu C and Yang PX: c-Jun NH2-terminal kinase 1/2 and endoplasmic reticulum stress as interdependent and reciprocal causation in diabetic embryopathy. Diabetes 62: 599-608, 2013.

26. Oakes SA and Papa FR: The role of endoplasmic reticulum stress in human pathology. Annu Rev Pathol 10: 173-194, 2015.

27. Cao SS, Luo KL and Shi L: Endoplasmic reticulum stress interacts with inflammation in human diseases. J Cell Physiol 231: 288-294, 2016

28. Mijošek V, Lasitschka F, Warth A, Zabeck H, Dalpke AH and Weitnauer M: Endoplasmic reticulum stress is a danger signal promoting innate inflammatory responses in bronchial epithelial cells. J Innate Immun 8: 464-478, 2016

29. Liu ZW, Zhu HT, Chen KL, Dong X, Wei J, Qiu C and Xue JH: Protein kinase RNA-like endoplasmic reticulum kinase (PERK) signaling pathway plays a major role in reactive oxygen species (ROS)-mediated endoplasmic reticulum stress-induced apoptosis in diabetic cardiomyopathy. Cardiovasc Diabetol 12: 158, 2013.

30. Ochoa CD, Wu RF and Terada LS: ROS signaling and ER stress in cardiovascular disease. Mol Aspects Med 63: 18-29, 2018.
31. Saco TV, Breitzig MT, Lockey RF and Kolliputi N: Epigenetics of mucus hypersecretion in chronic respiratory diseases. Am J Respir Cell Mol Biol 58: 299-309, 2018.

32. Peñaranda Fajardo NM, Meijer C and Kruyt FA: The endoplasmic reticulum stress/unfolded protein response in gliomagenesis, tumor progression and as a therapeutic target in glioblastoma. Biochem Pharmacol 118: 1-8, 2016.

33. Bright MD, Itzhak DN, Wardell CP, Morgan GJ and Davies FE: Cleavage of BLOC1S1 mRNA by IRE1 is sequence specific, temporally separate from XBP1 splicing, and dispensable for cell viability under acute endoplasmic reticulum stress. Mol Cell Biol 35: 2186-2202, 2015

34. Ron D and Walter P: Signal integration in the endoplasmic reticulum unfolded protein response. Nat Rev Mol Cell Biol 8: 519-529, 2007.

35. Jiang D, Niwa $M$ and Koong AC: Targeting the IRE1 $\alpha-X B P 1$ branch of the unfolded protein response in human diseases. Semin Cancer Biol 33: 48-56, 2015.

36. Cao SS and Kaufman RJ: Unfolded protein response. Curr Biol 22: R622-R626, 2012.

37. Hetz C: The unfolded protein response: Controlling cell fate decisions under ER stress and beyond. Nat Rev Mol Cell Biol 13: 89-102, 2012.

38. Wu R, Zhang QH, Lu YJ, Ren K and Yi GH: Involvement of the IRE1 $\alpha$-XBP1 pathway and XBP1s-dependent transcriptional reprogramming in metabolic diseases. DNA Cell Biol 34: 6-18, 2015.

39. Chen $C$ and Zhang X: IRE1 $\alpha$-XBP1 pathway promotes melanoma progression by regulating IL-6/STAT3 signaling. J Transl Med 15: 42, 2017.

40. Urano F, Bertolotti A and Ron D: IRE1 and efferent signaling from the endoplasmic reticulum. J Cell Sci 21: 3697-3702, 2000.

41. Park SH, Gong JH, Choi YJ, Kang MK, Kim YH and Kang YH: Kaempferol inhibits endoplasmic reticulum stress-associated mucus hypersecretion in airway epithelial cells and ovalbumin-sensitized mice. PLoS One 10. e0143526, 2015.

42. Iwakoshi NN, Lee AH, Vallabhajosyula P, Otipoby KL, Rajewsky K and Glimcher LH: Plasma cell differentiation and the unfolded protein response intersect at the transcription factor XBP1. Nat Immunol 4: 321-329, 2003.

43. Glimcher LH: XBP1: The last two decades. Ann Rheum Dis 69 (Suppl 1): S67-S71, 2010.

44. Wang G, Xu Z, Wang R, Al-Hijji M, Salit J, Strulovici-Barel Y, Tilley AE, Mezey JG and Crystal RG: Genes associated with MUC5AC expression in small airway epithelium of human smokers and non-smokers. BMC Med Genomics 5: 21, 2012.

45. Yoshida H, Okada T, Haze K, Yanagi H, Yura T, Negishi M and Mori K: ATF6 activated by proteolysis binds in the presence of NF-Y (CBF) directly to the cis-acting element responsible for the mammalian unfolded protein response. Mol Cell Biol 20: 6755-6767, 2000.

46. Spaan CN, Smit WL, van Lidth de Jeude JF, Meijer BJ, Muncan V, van den Brink GR and Heijmans J: Expression of UPR effector proteins ATF6 and XBP1 reduce colorectal cancer cell proliferation and stemness by activating PERK signaling. Cell Death Dis 10: 490, 2019.

47. Gonen N, Sabath N, Burge CB and Shalgi R: Widespread PERK-dependent repression of ER targets in response to ER stress. Sci Rep 9: 4330, 2019.

This work is licensed under a Creative Commons Attribution-NonCommercial-NoDerivatives 4.0 International (CC BY-NC-ND 4.0) License. 